\title{
An Evaluation Of Infra-Red Tympanic Thermometry For Thermal Physiology Research
}

\author{
Maj MCM Bricknell \\ BM, MMedSci, MRCGP, AFOM, DRCOG, DFFP, RAMC
}

23 Parachute Field Ambulance, Normandy Barracks, Montgomery Lines, Aldershot, Hants GU11 $2 A X$

SUMMARY: This study forms part of a research programme to investigate the relationships between the rise inco core temperature and the environmental temperature in soldiers exercising at a constant rate. The measurement of core temperature is a fundamental requirement for this research. The aim of this study was to evaluate the use of an infra-red tympanic thermometer (IVAC Corecheck ${ }^{\mathrm{TM}}$ ) for use in thermal physiological studies conducted outside ${ }^{\mathcal{S}}$ the laboratory environment by comparing the right ear temperature by TM thermometer and the left ear externalo auditory meatus by thermistor. The slope of the regression line between the two measurements was 0.9967 with a correlation coefficient of 0.706 which is reasonable. The TM thermometer in general reads higher than the aurat $\omega_{-}$ thermometer with a $95 \%$ confidence limit for agreement from +0.78 to $-0.53^{\circ} \mathrm{C}$ of the aural temperature. Thus this= study demonstrated that the tympanic thermometer (IVAC Corecheck ${ }^{\mathrm{TM}}$ ) is sufficiently reliable compared to the aural thermistor to justify field trials.

\section{Introduction}

Casualties from heat illness are an important cause of morbidity in the Armed Forces. It was proposed to undertake some field studies to investigate the relationship between the change in core temperature and environmental temperature on soldiers exercising at a constant rate. This research programme depended on the identification of a device to measure body core temperature in the experimental subjects.

The measurement of central core temperature is a fundamental requirement of thermal physiology research. An ideal site to measure core temperature would be convenient, independent of environmental conditions and reliable. The measurement should reliably correlate with an accepted standard (usually pulmonary artery temperature). Unfortunately there is no such ideal solution. The core temperature may be measured experimentally in the oesophagus, rectum, mouth, external auditory meatus (EAM) and the tympanic membrane (TM). These are compared in ANNEX A.

The infra-red tympanic membrane (TM) temperature would seem to be a suitable method for measuring the core temperature of a large number of experimental subjects in studies conducted outside the laboratory environment. There are several studies that have shown correlations between the TM temperature reading and oral, oesophageal, rectal and pulmonary artery temperatures $(1,2,3,4)$. It has not been possible to find any reported studies comparing TM temperature and external auditory canal measurements.

It was proposed to use an IVAC Corecheck ${ }^{\mathrm{TM}}$ tympanic thermometer to collect core temperature measurements in the field before and after exercise. The IVAC Corecheck ${ }^{\mathrm{TM}}$ tympanic thermometer measures IR radiation emitted from the tympanic membrane. A plastic cover is stretched over the head of the device from a specially designed dispenser. The probe is then inserted into the external auditory meatus using firm pressure. The probe is aimed towards the patient's opposite eye to ensure a 'good view' of the tympanic membrane. The trigger switch is pressed and released. Afterc approximately 3 seconds a temperature reading appears oni the display. Once the reading has been taken the probe is ${ }^{\AA}$ removed from the patient's ear and the probe covers removed. The thermometer is now reset and anothertemperature may be taken. The specifications for the IV flo Corecheck $^{\mathrm{TM}}$ thermometer are shown Table 1.

Table 1

Specifications for the IVAC Corecheck ${ }^{\mathrm{TM}}$ Thermometer

\begin{tabular}{|c|c|}
\hline Measurement range: & $25^{\circ} \mathrm{C}$ to $43.3^{\circ} \mathrm{C}$ \\
\hline Accuracy: & $\pm 0.1^{\circ} \mathrm{C}$ \\
\hline Response time: & $<3 \operatorname{secs}$ \\
\hline Operating range: & $15.6^{\circ} \mathrm{C}-43.3^{\circ} \mathrm{C}$ \\
\hline
\end{tabular}

The aim of this study was to evaluate the use of an infra-? red tympanic thermometer (IVAC Corecheck ${ }^{\mathrm{TM}}$ ) for use ino thermal physiological studies conducted outside the laboratory environment by comparing the right ear temperature by $\mathrm{TM}$ thermometer and the left ear externa auditory meatus by thermistor.

\section{Method}

This study was conducted using the climatic chamber a? the Centre for Human Sciences, Defence Research Agency. Farnborough, Hants. The subjects were from two courses of medical officers who were involved in a trial to give them experience of working in a hot environment. This required each study subject to step on and off a 12 inch stool at the rate of 12 steps per minute for one hour. The environmentale. temperature was maintained at a Wet-Bulb Globeస Temperature Index of $36.5^{\circ} \mathrm{C}$ throughout the experiment The subjects' temperatures were monitored using an aura thermistor in the left ear insulated with cotton-wool and eardefenders. In addition each subject's heart rate wase 
monitored by ECG. Subjects could be withdrawn from the trial in the following circumstances: their own request, aural thermistor temperature $>38.5^{\circ} \mathrm{C}$, heart rate $>180$ beats per minute, or if the supervising doctor considered them to be unfit to continue. This protocol was approved by the Centre's ethics committee.

The IVAC Corecheck ${ }^{\mathrm{TM}}$ thermometer had been calibrated in accordance with the manufacturer's instructions. Measurements of the TM temperature were taken every 10 minutes until either completion of the trial or the subject dropped out. Each measurement was taken by the same investigator (MCMB) using the recommended technique including an 'aural tug' on the pinna to straighten the external auditory canal (5).

The subjects were fitted with the aural thermistors by specially trained staff and allowed 10 minutes for the instrument to equilibrate prior to entering the hot chamber. Measurements of external auditory canal temperature were taken automatically every 10 minutes until completion of the experiment.

\section{Results}

There were 23 subjects of whom 11 were withdrawn during the work-in-heat test because of raised core temperature or excessive pulse rate. The results have been analysed using the statistical techniques for assessing agreement between two methods of clinical measurement $(6,7,8)$. One hundred and two comparison readings were taken with a mean difference between TM temperature and aural temperature of $0.123^{\circ} \mathrm{C}$ (standard deviation 0.335 ). The $95 \%$ confidence limits for agreement are +0.78 to $0.53^{\circ} \mathrm{C}$. Figure 1 shows the TM temperature plotted against the aural temperature.

The difference between the two measurements demonstrates the bias between the two methods. As the true core temperature is unknown the best estimate would be the mean of the TM and aural temperature. If the bias is plotted against the mean of the two values for each occasion the measurements were taken, the graph at Figure 2 can be created. It can be seen that the difference between the two measuring techniques reduces as the core temperature increases. The linear trendline is also plotted with parallel limit lines at +/- 1.96SD.

\section{Discussion}

The IVAC Corecheck ${ }^{\mathrm{TM}}$ thermometer offers substantial advantages over other methods of measuring core temperature for field physiology studies. There is not a suitable alternative that can be used in studies involving many subjects who cannot be connected to electronic transducers or receiving equipment. Previous work has demonstrated that the infra-red thermometer may be useful in clinical practice for individual patients, particularly children (1-5).

Figure 1 shows that the slope of the regression line between the two measurements is 0.9967 with a correlation coefficient of 0.706 which is reasonable. The TM thermometer in general reads higher than the aural thermometer with a 95\% confidence limit for agreement from +0.78 to $-0.53^{\circ} \mathrm{C}$ of the aural temperature. Figure 2 shows the difference in temperature (bias) between the two thermometers plotted. Thus the TM thermometer should detect the majority of clinically significant episodes of hyperthermia if the threshold for diagnosis has a safety margin of $0.5^{\circ} \mathrm{C}$ particularly as the difference between the two measurements reduces as the measured temperature rises.

The measured TM temperature may vary from the true TM temperature for the reasons listed in Table 2. The subjects' ear canals were not inspected prior to instrumentation and thus it is possible that the results from some subjects were unreliable. However in clinical practice the ear canal is not routinely inspected prior to measuring the tympanic temperature. As the left ear canal was obscured by the aural thermistor it was not possible to measure both sides concurrently which would have tested the reproducibility of the measurements. Similarly single measurements were taken because only one thermometer was available for the Study. Multiple measurements would have enabled an estimate of repeatability to be made.

\section{Reasons for the Measured TM Temperature Differing from the True TM Temperature}

1. Sensor not facing the tympanic membrane.

2. Sensor window dirty or damaged.

3. Thermometer requiring calibration.

4. Sensors 'view' obscured by wax or narrow ear canal.

\section{Conclusion}

This study compared the use of a IVAC Corecheck ${ }^{\mathrm{TM}}$. thermometer to measure changes in core temperature with aural thermistors which is a recognised non-invasive technique in use at the Centre for Human Sciences, Defence Research Agency. Neither method is ideal. However both are suitable if consent for rectal thermometers is likely to be withheld. If subjects cannot be instrumented then the IVAC Corecheck ${ }^{\mathrm{TM}}$ thermometer offers a rapid method for measuring core temperature.

The proposed protocol for the field study was based on the measurement of multiple subjects and comparing the change in group mean core temperature as a result of exercise in heat. The precision of the temperature measuring device in this design is less critical than that required for a study involving single subjects because of the 'smoothing' effect of pooling results.

The results show that the core temperature measured by the IVAC Corecheck ${ }^{\mathrm{TM}}$ thermometer is sufficiently reliable compared to the aural thermistor to justify further studies. These should include an assessment of repeatability of measurements from one ear, and reproducibility of measurements by comparing measurements taken concurrently from both ears. In addition a study should be undertaken to determine if the mean TM temperature of a group of subjects is affected by ambient temperature. 
Bricknell

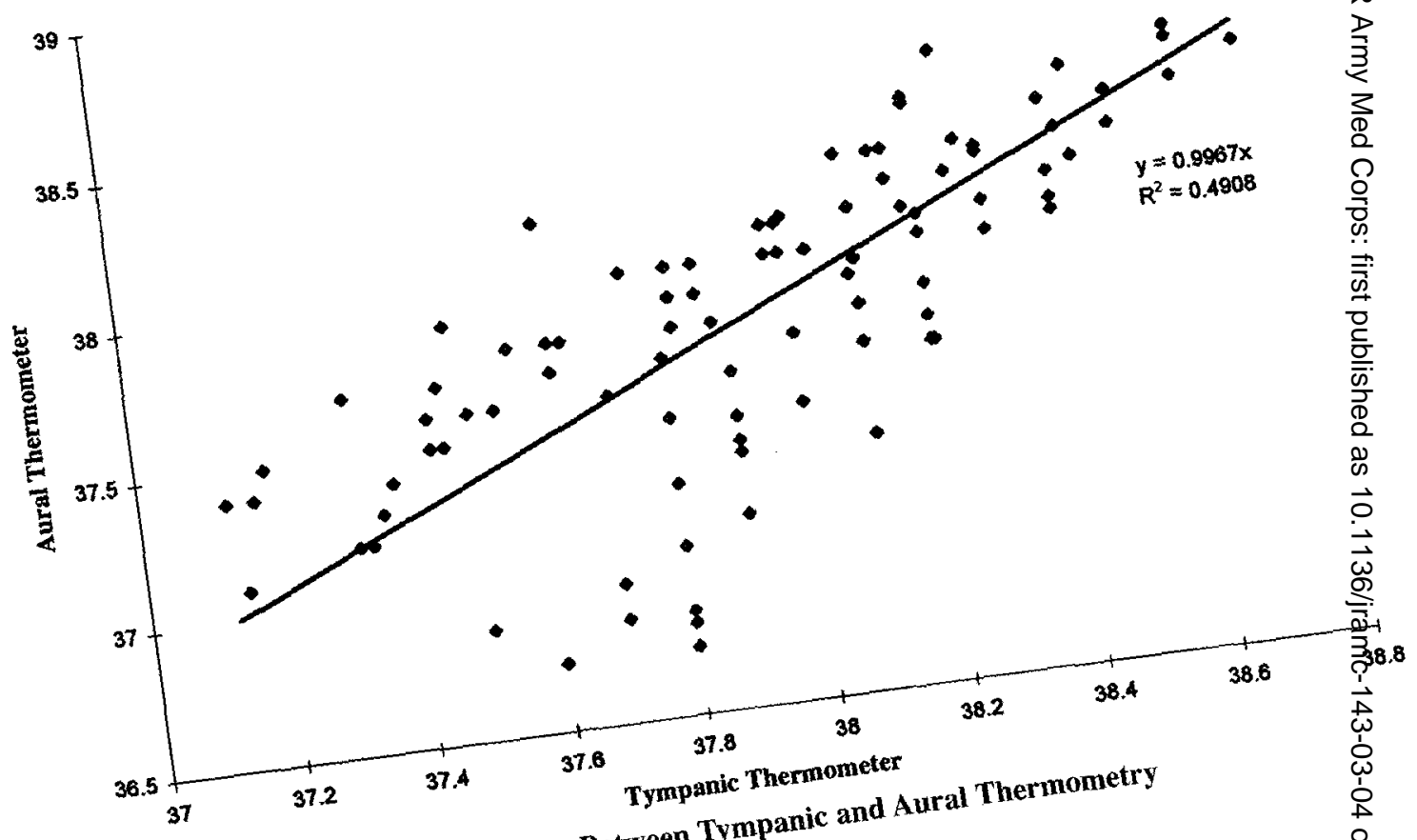

Fig 1. Correlation Between Tympanic and Aural Therma

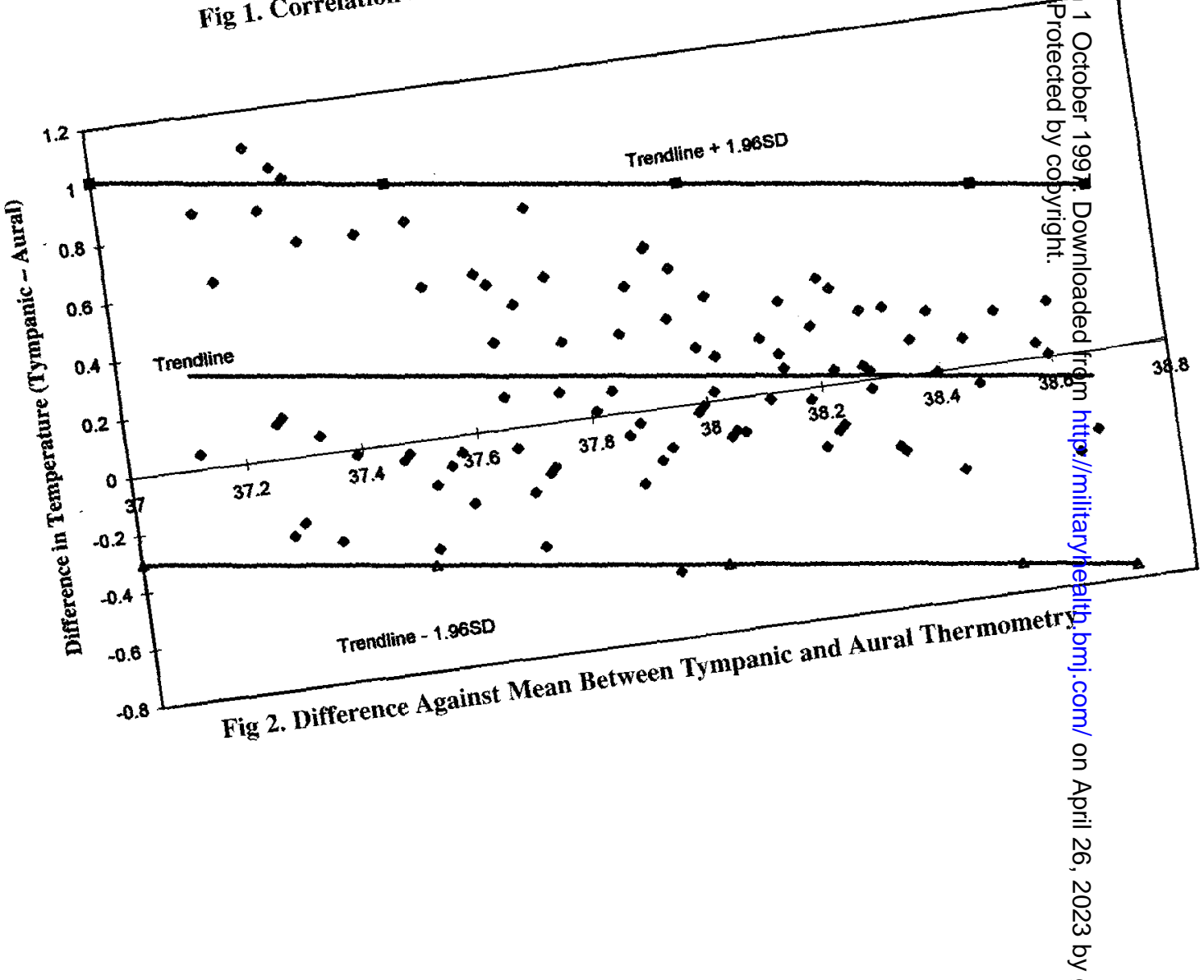




\section{ANNEX A \\ Comparison of Methods of Measuring Body Temperature}

Oesophageal temperature

This method involves the insertion of a catheter containing an electronic thermometer through the nose into the oesophagus. This is advanced until the tip lies at the level of the left atrium. This site is approximately iso-thermal with the blood in the left atrium. This is an invasive method and the insertion of the catheter may be uncomfortable. Each subject requires individual instrumentation. The subject must avoid swallowing saliva or this will lower the recorded temperature. Rectal temperature

The rectal temperature may be measured using a mercury-in-glass thermometer or an electric temperature sensor. The mercury-in-glass thermometer is inappropriate for experiments in which the subjects are exercising. This is an invasive technique that also requires individual instrumentation. Furthermore there is a delay before rectal temperature equilibrates with the core temperature thus reducing its value in thermal physiological experiments.

Oral temperature

This is the measurement that is most frequently used in clinical practice. The temperature may be measured using mercury-in-glass or electric thermometers. There is a variable delay between 3-10 minutes to reach a stable measured temperature. This method cannot be used during exercise because the subject must not breathe through the mouth during the measurement.

External auditory meatus (EAM) temperature

A temperature sensor within an ear plug is placed inside the external auditory meatus. This device is then thermally insulated by additional padding. This is an invasive method, again requiring individual instrumentation. The recorded temperature may differ from the 'core' temperature due to environmental conditions affecting the temperature of the facial skin. In spite of these limitations this technique is frequently used in physiological studies.

Tympanic membrane temperature

The tympanic membrane is perfused by blood from the carotid arteries and so this temperature should reflect core temperature. This measurement should be less sensitive to environmental conditions than the EAM temperature. The original technique involved the placing of a sensor against the tympanic membrane. This was occasionally uncomfortable and there were reports of accidental perforation of the tympanic membrane. However is now possible to measure the infras red (IR) energy emitted from the tympanic membrane using an IR detector. This is a quick (less than 3 seconds) non? invasive method. It does not require individual instrumentation.

Telemetry pill systems

Telemetry systems based on swallowing a radio-transducer linked to a thermometer offers the ideal solution. Howeve the mobility of the pill inside the intestines can cause variation in the measured temperature. Furthermore this is an? expensive method.

\section{REFERENCES}

1. TERNDRUP TE. An appraisal of temperature assessment by infra-red emission detection tympanic thermometry. Ann Emerg Med 1992; 21: 1483-1492.

2. FLo G, BROWN M. Comparing three methods of temperature taking: oral mercury-in-glass, oral Diatek and tympanic. First Temp Nursing Res 1995; 44: 120122.

3. ERICKSON RS, MEYER TM. Accuracy of infra-red ear thermometry and other temperature methods in adults. Am J Crit Care 1994; 3: 40-54.

4. JAKOBSSON J, Nilsson A, CARLSSON L. Core temperature measured in the auricular canal: comparison between four different tympanic thermometers. Acta Anaesthesiol Scand 1992; 36: 819-
824.

5. TERNDRUP TE, RAIK J. Impact of operator technique and device on infra-red emission detection tympanic thermometry. J Emerg Med 1992; 10: 683-687.

6. Bland JM, Altman DG. Statistical methods for assessing agreement between two methods of clinical measurement. Lancet 1986; I: 307-10.

7. BROWN RA, Swanson Beck J. Statistics on microcomputers: a non-algebraic guide to their appropriate use in biomedical research and pathology laboratory practice. 4 Correlation and regression. $J$ Clin Pathol 1989; 42: 4-12.

8. BRenNan P, Silman A. Statistical methods for assessing observer variability in clinical measures. $\mathrm{Br}$ Med J 1992; 304: 1491-4. 